\title{
Hadron production and $\Upsilon$ suppression at the LHC
}

\author{
Georg Wolschin ${ }^{1, a}$ \\ ${ }^{1}$ Institut für Theoretische Physik der Universität Heidelberg, Philosophenweg 16, D-69120 Heidelberg, \\ Germany, EU
}

\begin{abstract}
Hadron production in relativistic heavy-ion collisions at LHC energies is investigated. After a brief consideration of stopping, particle production is accounted for in a nonequilibrium-statistical model with two fragmentation sources, and a central source that is mostly due to low- $x$ gluon-gluon collisions. The particle content and energy dependence of the three sources is discussed for $\mathrm{AuAu}$ (RHIC) and $\mathrm{PbPb}$ (LHC). Results for asymmetric $p \mathrm{~Pb}$ collisions are compared with ALICE and preliminary ATLAS data. The suppression of $\Upsilon$-mesons in the hot quark-gluon medium in $\mathrm{PbPb}$ collisions as compared to $p p$ at $\sqrt{s_{N N}}=2.76 \mathrm{TeV}$ is accounted for in a model that encompasses gluodissociation, collisional damping, screening, and reduced feed-down. Results are compared with CMS and ALICE data.
\end{abstract}

\section{Introduction}

A brief survey of several features of hadron production in relativistic heavy-ion collisions at LHC energies is given in this article, which is essentially based on results published in [1], and obtained in $[2,3]$ for charged-hadron production, and in the references cited in the text for bottomia suppression in $\mathrm{PbPb}$ collisions at $\mathrm{LHC}$ energies.

The stopping process in the very first phase of the collision is mostly characterized by the interaction of fast valence quarks with low- $x$ gluons in the respective other nucleus. The fragmentation peaks in net-proton (proton - antiproton) rapidity distributions are clearly visible in SPS and RHIC data [4-7]. When considering rapidity or pseudorapidity distributions of produced charged particles, the fragmentation distributions also play a significant role, but since they shift to large values of rapidity at LHC, particle production near mid rapidity is almost exclusively determined by the central low- $x$ gluon-gluon source.

This source cancels out in net-proton distributions, but in charged-hadron distributions its importance rises strongly with incident energy. In this note the relative importance of the three sources is investigated as function of energy from a direct comparison of the analytical solutions of a nonequilibrium-statistical relativistic diffusion model (RDM) [8] with PHOBOS [9] and recent ALICE data [10] data. This allows also for predictions at the LHC design energy, and for an analysis of the asymmetric $p \mathrm{~Pb}$ system at the current LHC energy of $5.02 \mathrm{TeV}$.

\footnotetext{
a e-mail: wolschin@uni-hd.de
} 


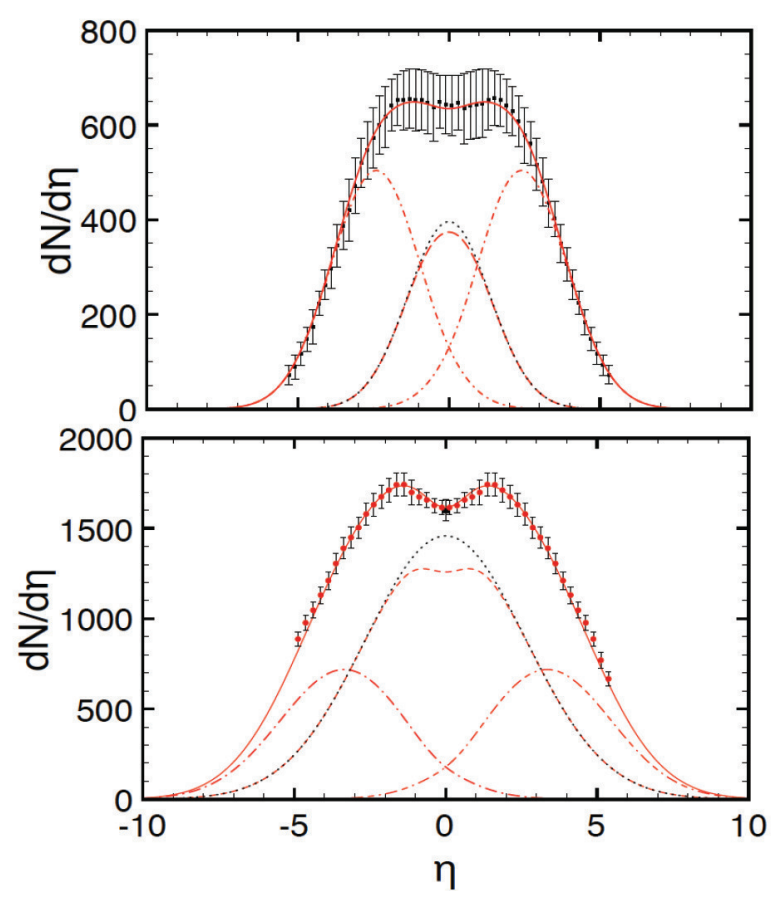

Figure 1. The RDM pseudorapidity distribution functions for charged hadrons in central $200 \mathrm{GeV} \mathrm{AuAu}$ (top frame) and 2.76 TeV PbPb collisions are adjusted through $\chi^{2}$-minimizations to the PHOBOS [9] (see also [11]) and ALICE [10] data. The underlying particle production sources are shown: dash-dotted curves are the fragmentation sources, dashed curves the mid rapidity gluon-gluon sources, and dotted curves the central sources without the effect of the Jacobian transformation from rapidity to pseudorapidity. The particle content in the gluon-gluon source rises strongly with increasing c.m. energy. From [3]

\section{Hadron production sources}

The relativistic diffusion model (RDM) for the investigation of the time evolution of particle production sources in relativistic heavy-ion collisions has been applied in [1] to charged-hadron production in $\mathrm{AuAu}$ collisions at RHIC energies, and to $\mathrm{PbPb}$ collisions at LHC energies of $2.76 \mathrm{TeV}$. The sizes of the fragmentation sources, and of the mid rapidity gluon-gluon source have been identified as functions of centrality and energy. The results for central AuAu collisions at $200 \mathrm{GeV}$, and central $\mathrm{PbPb}$ collision at $2.76 \mathrm{TeV}$ are shown in figure 1, together with the underlying particle production sources.

The particle content in the low- $x$ gluon-gluon source is seen to rise strongly with increasing c.m. energy, and constitutes the largest particle production source at LHC energies. The effect of the Jacobian transformation from rapidity $y$ to pseudorapidity $\eta$ - which acts mostly on the central source - is also shown. It causes a dip in the mid-rapidity source, but has a rather negligeable effect on the fragmentation sources at larger values of rapidity. The incoherent superposition of the sources gives a good representation of the ALICE data [10], and the five RDM parameters can be determined in a $\chi^{2}$-minimization with respect to these data [1]. The minimum near mid rapidity occurs due to both, the effect of the Jacobian plus the interplay of fragmentation and central sources. 


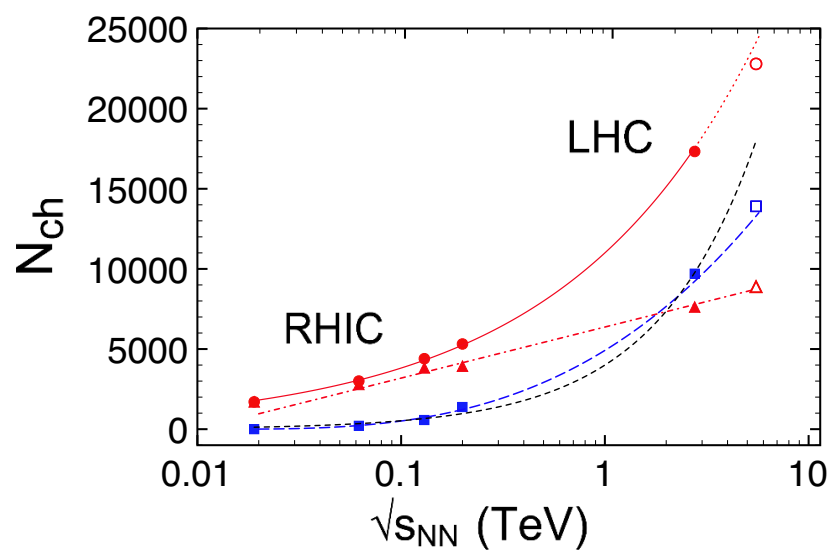

Figure 2. Number of produced charged hadrons as function of the c.m. energy $\sqrt{s_{N N}}$ from RDM-fits of the available data for central heavy-ion collisions at 0.019, 0.062, 0.13, 0.2 TeV (RHIC, AuAu), 2.76 TeV (LHC, $\mathrm{PbPb}$ ), plus extrapolation to $5.52 \mathrm{TeV}$. Circles are the total numbers, following a power law $\propto s_{N N}^{0.23}$. Triangles are particles from the fragmentation sources $\propto \log \left(s_{N N} / s_{0}\right)$. Squares are hadrons produced from the midrapidity source, with a dependence $\propto \log ^{3}\left(s_{N N} / s_{0}\right)$. A power law $\propto s_{N N}^{0.44}$ [1] is also shown, but fails to fit the extrapolated $5.52 \mathrm{TeV}$ yield. The gluon-gluon source (dashed) becomes the main source of particle production between RHIC and LHC energies. From [3].

The particle contents of the sources are displayed in figure 2, which resembles the analogous figure in [1], but differs in a decisive detail. The total particle content is found to follow a power law $[1,3]$

$$
N_{c h}^{t o t}=1.1 \cdot 10^{4}\left(s_{N N} / s_{0}\right)^{0.23}
$$

with $s_{0}=1 \mathrm{TeV}^{2}$, whereas the particle content in the two fragmentation sources is as expected a logarithmic function of the energy

$$
N_{c h}^{q g}=695 \cdot \ln \left(s_{N N} / s_{0}\right)
$$

with $s_{0}=100 \mathrm{GeV}^{2}$. The midrapidity gluon-gluon source is approximated by the thin dashed line following a power law as was already proposed in [1]

$$
N_{c h}^{g g} \simeq 4 \cdot 10^{3}\left(s_{N N} / s_{0}\right)^{0.44}
$$

with $s_{0}=1 \mathrm{TeV}^{2}$. However, when considering also the yield predicted within the relativistic diffusion model (RDM) for the LHC design energy of $5.52 \mathrm{TeV}$, the power law fails to fit the expected yield, whereas a cubic log dependence agrees with the prediction,

$$
N_{c h}^{g g}=7.5 \cdot \ln ^{3}\left(s_{N N} / s_{0}\right)
$$

where $s_{0}=169 \mathrm{GeV}^{2}[3]$.

It remains to be seen whether the data actually follow the model prediction. In the upcoming $\mathrm{PbPb}$ run at the LHC in 2015, the c.m. energy is scheduled to be $5.125 \mathrm{TeV}$, corresponding to $13 \mathrm{TeV} p p$. 


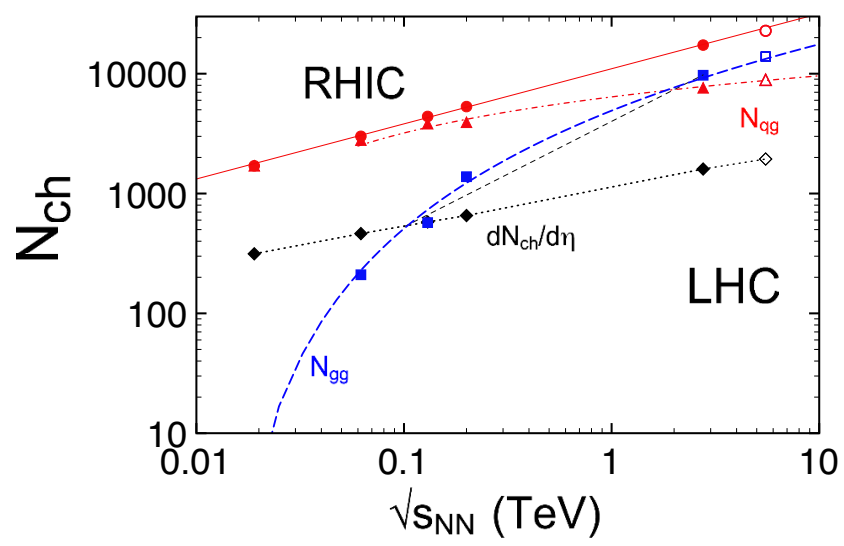

Figure 3. The total charged-hadron production in central $\mathrm{AuAu}$ and $\mathrm{PbPb}$ collision in the energy region 19.6 $\mathrm{GeV}$ to $5.52 \mathrm{TeV}$ is following a power law $N_{\text {tot }} \propto\left(s_{N N} / s_{0}\right)^{0.23}$ (solid line), whereas the particle content in the fragmentation sources is $N q g \propto \ln \left(s_{N N} / s_{0}\right)$, dash-dotted curve. The particle content in the mid-rapidity source obeys $N_{g q} \propto \ln ^{3}\left(s_{N N} / s_{0}\right)$, dashed curve, not too far from a power law (short-dashed line) only in the intermediate energy range $0.1-2.76 \mathrm{TeV}$. The energy dependence of the mid rapidity yield is shown as a dotted line. From [3].

The total charged-hadron yield predicted by Eq. (2) at this energy is $N_{c h}^{t o t}=23,327$, with the central source contributing $N_{c h}^{g g}=12,811$ charged hadrons according to Eq. (4).

When examining the RDM results for the particle content of the sources more closely also in the low-energy region where RHIC data are available, it turns out that the power law Eq. (3) is an acceptable approximation to $N_{c h}^{g g}$ only between about $100 \mathrm{GeV}$ and $2.76 \mathrm{TeV}$. This becomes particularly obvious in figure 3 , where the same plot is shown using a double-logarithmic scale, following a suggestion by Trainor [14]. The cubic-log dependence of the gluon-gluon source (dashed) is seen to fit the points that have been extracted from my RDM-analyses [1] of PHOBOS and ALICE data rather precisely at the available energies, and it agrees with the RDM-prediction at the LHC design energy of $5.52 \mathrm{TeV}$.

As required by the RDM analysis of the $19.6 \mathrm{GeV}$ AuAu data, the gluon-gluon contribution becomes unimportant below $20 \mathrm{GeV}$ - whereas a power law would still predict a yield of about 100 charged hadrons per unit of rapidity in this energy region. Although a hybrid function with a logdependence at RHIC energies that turns into a power law at LHC energies may appear as a reasonable compromise $[15,16]$, the $\ln ^{3}$-dependence for the central source offers a more precise fit of the RDMresults. A more detailed discussion of this behaviour, and its underlying physical reason is given in [3].

\section{Asymmetric systems}

With the same approach, also asymmetric systems such as $p \mathrm{~Pb}$ at LHC energies can be investigated $[1,2]$. Here the fragmentation sources have unequal particle content, and the midrapidity source is not centered at $\langle\eta\rangle=0$, but at a centrality- and transverse momentum-dependent equilibrium value of the rapidity. The result for minimum-bias $p \mathrm{~Pb}$ collisions at the LHC energy of $5.02 \mathrm{TeV}$ is shown $[1,2]$ in figure 4 in a $\chi^{2}$-minimization with respect to ALICE data [13], and preliminary ATLAS data [12]. 


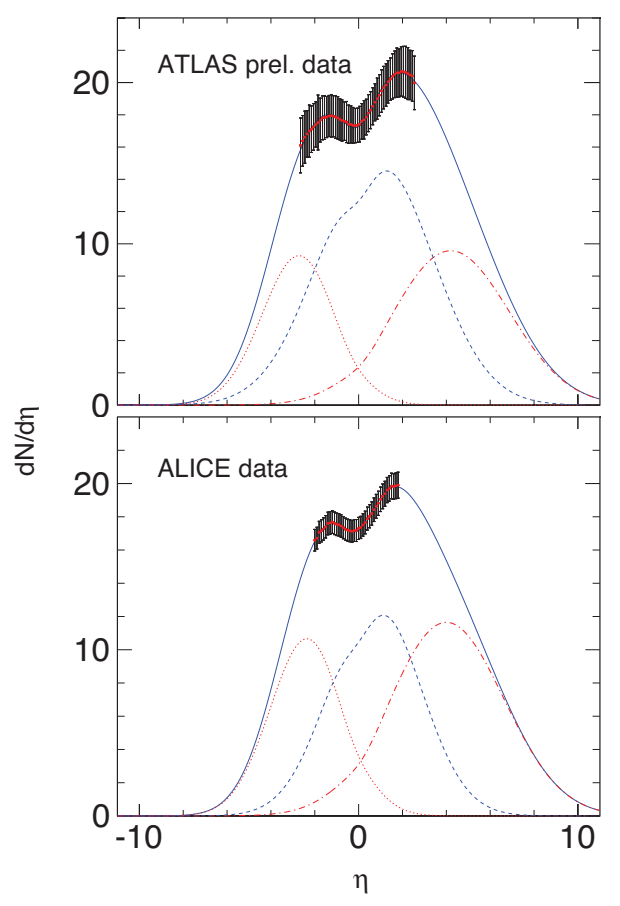

Figure 4. The predicted RDM pseudorapidity distribution functions for charged hadrons in minimum bias $p \mathrm{~Pb}$ collisions at LHC c.m. energy of $5.02 \mathrm{TeV}$ shown here are adjusted in the mid-rapidity region to the preliminary ATLAS (top) [12], and published ALICE (bottom) data [13] (systematic error bars only for ALICE). The measured preliminary ATLAS data $(0-90 \%)$ have been scaled by the geometric cross-section ratio $\sigma(0-90 \%) / \sigma(0-100 \%) \simeq 0.898$ to make them directly comparable to the minimum-bias ALICE results. The underlying distributions in the three-sources RDM are also shown. From [1,2].

The RDM-analysis of $p \mathrm{~Pb}$ collisions at LHC energies reveals excellent agreement with the preliminary centrality-dependent data taken by both, the ALICE and the ATLAS collaborations. We have determined the RDM-parameters in $\chi^{2}-$ minimizations with respect to these data. At all centralities, the mid-rapidity source has the largest particle content, but the fragmentation sources are necessary for a detailed understanding of the centrality-dependent shape of the total distribution functions. It is the interplay of the three sub-distributions together with the effect of the Jacobian transformation that determines the pseudorapidity density distribution of produced charged hadrons.

The shapes of the total distribution functions indicate that the system has not reached statistical equilibrium. In particular, the centres of the fragmentation distributions remain far from the equilibrium values $y_{e q}(b)$ : It is not just the produced-particle yields that determine whether the emitting source is in statistical equilibrium, but rather the shapes of the distribution functions, which clearly deviate from equilibrium distributions - although local equilibrium in the hydrodynamic sense appears to be achieved for events with $p_{T} \leq 6-8 \mathrm{GeV} / c$ as may be inferred from the success of hydrodynamics to describe bulk properties. 


\section{$4 \Upsilon$ suppression in $\mathrm{PbPb}$ collisions at $\mathrm{LHC}$ energies}

The production of heavy mesons and in particular, of bottomia in initial hard partonic interactions in relativistic $\mathrm{PbPb}$ collisions at $\mathrm{LHC}$ energies is of special interest, because quarkonia in the hot fireball can act as a probe to test the properties of the hot medium. In our model $[17,18]$ we investigate the suppression of the $\Upsilon(n S)$ states in $\mathrm{PbPb}$ as compared to the expectation from scaled $p p$ collisions at the same energy, and compare with centrality-dependent CMS data [19] for the $1 S$ and $2 S$ states. In the hot medium, the three most important dissociation mechanisms are taken to be gluodissociation, damping, and screening of the real part of the quark-antiquark potential. The calculated gluodissociation cross section for the $1 S$ and $2 S$ states together with the thermal gluon distribution function are shown as functions of the gluon energy at two different temperatures in figure 5.

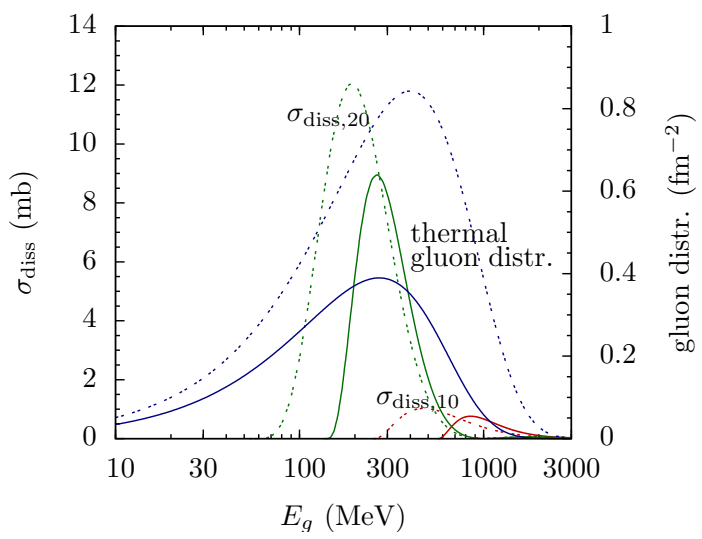

Figure 5. Gluodissociation cross section $\sigma_{\text {diss }}$ (left scale) of the $\Upsilon(1 S)$ and $\Upsilon(2 S)$ states and the thermal gluon distribution (right scale) plotted for temperature $T=170$ (solid curves) and $250 \mathrm{MeV}$ (dotted curves) as functions of the gluon energy $E_{g}$. From Nendzig and Wolschin [18].

Since the matrix elements that characterize gluodissociation (see formulae in [18]) have a finite extent in the gluon energy space, the corresponding cross sections exhibit maxima. Still, when averaged over the temperature-dependent gluon-energy distribution, and considering the momentum-dependent running of the strong-coupling constant, the average gluodissociation cross section rises monotonically as function of temperature in the region of interest - that is, for initial temperatures of $T \simeq 550$ $\mathrm{MeV}$ at the $\Upsilon$ formation time $\tau_{F} \simeq 0.1-0.5 \mathrm{fm} / c[18]$.

In addition to gluodissociation in the hot medium, we have also considered the damping widths due to the imaginary part of the quark-antiquark potential, and the screening of the real part. Gluodissociation and damping widths add up incoherently to total bottomia widths for the six states considered in the hot fireball, namely, $\Upsilon(1 S, 2 S, 3 S)$ and $\chi_{b}(1 P, 2 P, 3 P)$; other spin-triplet states are above the $B \bar{B}$ threshold and hence, are not relevant for a comparison with the measured $\Upsilon(n S)$ suppression.

The time and centrality dependence of the suppression of these six states in the hot medium is then calculated based on an ideal hydrodynamic expansion of the medium that includes transverse expansion, as well as the relativistic Doppler effect whenever the mean transverse momenta of the produced bottomia states and the $p_{T}$ of the expanding medium are different from each other. At the end of the QGP phase - when the temperature has dropped below the critical temperature - we calculate the centrality-dependent QGP suppression factors $R_{A A}^{Q G P}$.

It is very relevant to include at all impact parameters the corona zone where the temperature never rises above the critical value so that none of the QGP dissociation mechanisms play a role there, leading to an occupation of the excited states even in more central collisions where the excited states melt in the high-temperature region due to screening. In the corona, additional hadronic suppression that is mostly due to collisions with pions - which we have investigated separately - may occur [20]. 


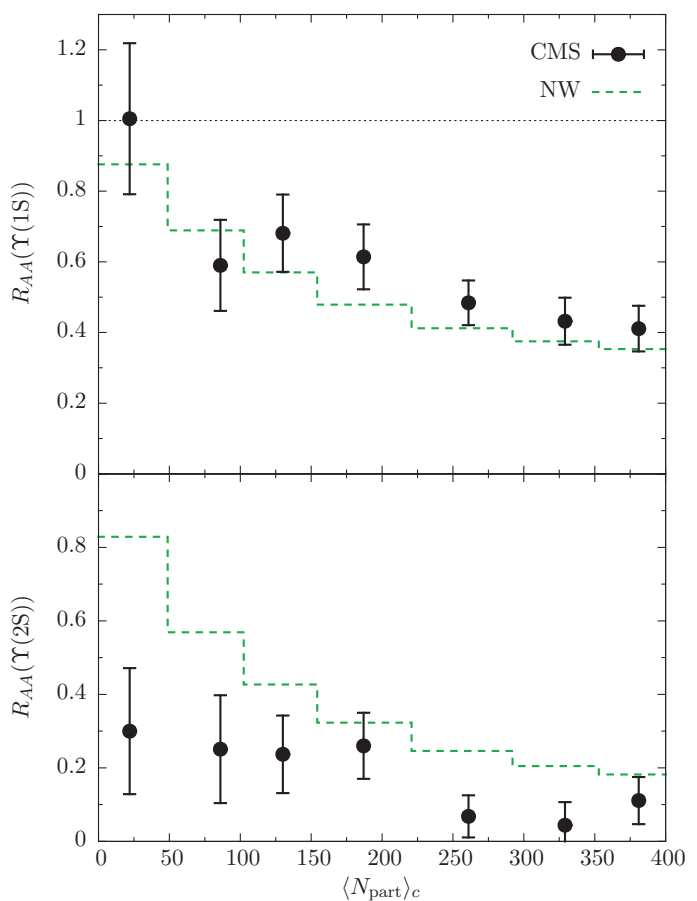

Figure 6. $\Upsilon(n S)$ suppression factors in $2.76 \mathrm{TeV}$ $\mathrm{PbPb}$ measured by CMS [19] (circles) and calculated with the model of Nendzig and Wolschin [18] (dashed lines) for $\tau_{\text {init }}=0.1 \mathrm{fm} / c, \tau_{F}=0.3 \mathrm{fm} / c, T_{i}=550$ $\mathrm{MeV}$ and $T_{c}=170 \mathrm{MeV}$, averaged over transverse momentum, $4 \mathrm{GeV} / c<p_{T}<24 \mathrm{GeV} / c$. From [20].

Once the centrality-dependent suppression in the hot fireball and in the corona is established, the bottomia states de-excite in a feed-down cascade before being detected through the emission of $\mu^{+} \mu^{-}$pairs, with a branching ratio of approximately $2.48 \%$ for the $1 S$ state, $1.93 \%$ for the $2 S$ state, and $2.18 \%$ for the $3 S$ state. The calculation of the feed-down cascade including the newly detected $\chi_{b}(3 P)$ state is described in [21]. It yields the final centrality-dependent suppression factors $R_{A A}$ [18] as shown in figure 6 in comparison with the CMS data [19] for the $\Upsilon(1 S)$ and $\Upsilon(2 S)$ states, with a parameter set indicated in the caption.

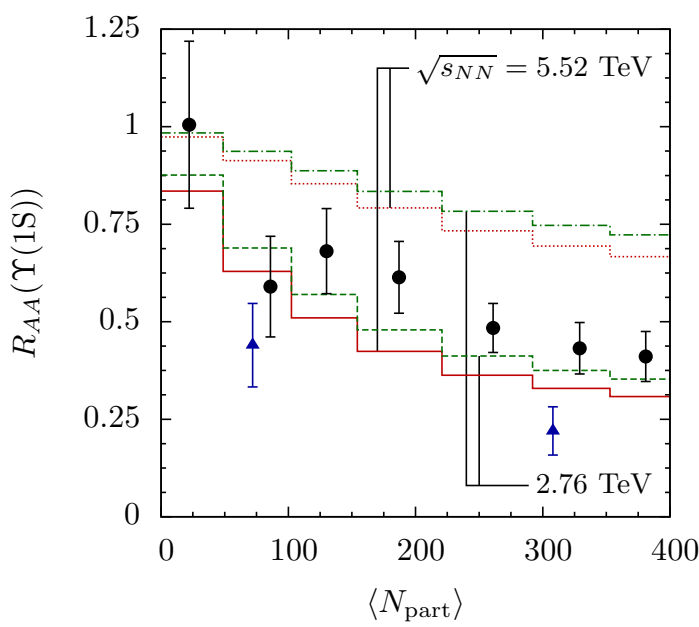

Figure 7. Predictions for $R_{A A}(1 S)$ and $R_{A A}^{Q G P}(1 S)$ for $\mathrm{PbPb}$ collisions at $\sqrt{s_{N N}}=5.52 \mathrm{TeV}$ (solid and dotted lines, respectively) and the previous results for $\sqrt{s_{N N}}=2.76 \mathrm{TeV}$ (dashed and dash-dotted lines, respectively) as a function of centrality, averaged over $p_{T}$, plotted for an $\Upsilon$ formation time of $\tau_{F}=0.3 \mathrm{fm} / c$, together with data from CMS [19] and ALICE [22] for $\sqrt{s_{N N}}=2.76 \mathrm{TeV}$. From [18]. 
The suppression of the spin-triplet ground state and its centrality dependence is in good agreement with the data for all centralities except for the $40-50 \%$ bin. For the first excited $2 S$ state, however, the calculated centrality dependence is too steep. Although the overall amount of suppression would be enhanced through a shorter formation time $\tau_{F}$ with ensuing larger QGP lifetime, such that the central bins are better accounted for, the problem of missing suppression in the three peripheral bins $50-100 \%, 40-50 \%$ and 30-40\% remains to be solved. We have checked the influence of hadroninduced dissociation in the corona region, with the result that the centrality-dependence of pioninduced dissociation is probably not strong enough to account for the difference. Another interesting possibility may be the dissociation through the transient magnetic fields, which is expected to be more pronounced for peripheral collisions.

Since the results for the ground-state suppression are in agreement with experiment, we have also calculated the $\Upsilon(1 S)$ suppression in $\mathrm{PbPb}$ at the LHC design energy of $5.52 \mathrm{TeV}$, solid line in figure 7. The central initial temperature is increased by $6.6 \%$ to $586 \mathrm{MeV}$ using the scaling relation between the initial entropy density and the charged-particle multiplicity density $s_{0} \propto d N_{c h} / d \eta \propto T_{0}^{3}$. The ground state is found to be slightly more suppressed in the medium at the higher energy and hence, the total suppression in $\mathrm{PbPb}$ as compared to $p p$ is stronger. However, the effect is less than $10 \%$ when doubling the c.m. energy.

\section{Conclusion}

Particle production sources in relativistic heavy-ion collisions at RHIC and LHC energies have been investigated. The relevance and effect of the fragmentation sources not only in stopping, but also in particle production has been outlined, and discussed as function of incident energy. The particle content in the mid-rapidity gluon-gluon source increases rapidly (stronger than a power law) with incident c.m. energy. Charged-hadron production in the asymmetric $p \mathrm{~Pb}$ system at $5.02 \mathrm{TeV}$ is investigated accordingly.

The suppression of the strongly bound $\Upsilon(1 S)$ spin-triplet ground state in $\mathrm{PbPb}$ collisions at LHC energies as compared to scaled $p p$ collisions at the same energy is a sensitive indicator for the properties of the quark-gluon plasma that causes gluodissociation and damping. The feed-down cascade from the excited bottomia states to the ground state produces additional suppression, since the excited states melt through screening, or depopulate through dissociation processes. Our model results [18] for the ground state are in good agreement with the CMS data [19], but the suppression of the first excited state calls for additional centrality-dependent dissociation mechanisms.

\section{Acknowledgements}

I am much obliged to the organizers of ICNFP2014 - Larissa Bravina, Yiota Foka and Sonia Kabana - for setting up this interesting conference in the refreshing environment of OAC Kolymbari. The contributions of my students - in particular, Felix Nendzig and Johannes Hoelck - to the $\Upsilon$ dissociation project are gratefully acknowledged.

\section{References}

[1] G. Wolschin, J. Phys. G: Nuclear and Particle Physics 40, 45104 (2013)

[2] P. Schulz, G. Wolschin, submitted to J. Phys. G: Nuclear and Particle Physics (2014)

[3] G. Wolschin, submitted to Phys. Rev. C (2014)

[4] I.G. Bearden et al. (BRAHMS Collaboration), Phys. Rev. Lett. 93, 102301 (2004)

[5] H.H. Dalsgaard et al. (BRAHMS Collaboration), Int. J. Mod. Phys. E 16, 1813 (2007) 
[6] R. Debbie et al. (BRAHMS Collaboration), J. Phys. G 35, 104004 (2008)

[7] I.C. Arsene et al. (BRAHMS Collaboration), Phys. Lett. B 677, 267 (2009)

[8] G. Wolschin, Phys. Rev. C 69, 024906 (2004)

[9] B. Alver et al. (PHOBOS Collaboration), Phys. Rev. C 83, 024913 (2011)

[10] M. Guilbaud et al. (ALICE Collaboration), Nucl. Phys. A 904, 381c (2013)

[11] G. Wolschin, M. Biyajima, T. Mizoguchi, N. Suzuki, Annalen Phys. 15, 369 (2006)

[12] B. Cole et al. (ATLAS Collaboration), ATLAS-CONF-2013-096 p. to be published (2013)

[13] B. Abelev et al. (ALICE Collaboration), Phys. Rev. Lett. 110, 032301 (2013)

[14] T. Trainor, priv. comm. (2014)

[15] R. Sahoo, A. Mishra, Int. J. Mod. Phys. E 23, 1450024 (2014)

[16] R. Sahoo, A. Mishra, N. Behera, B. Nandi, submitted to AHEP, and arXiv:1408.5773 (2014)

[17] F. Nendzig, G. Wolschin, Phys. Rev. C 87, 024911 (2013)

[18] F. Nendzig, G. Wolschin, J. Phys. G: Nuclear and Particle Physics 41, 095003 (2014)

[19] S. Chatrchyan, V. Khachatryan, A.M. Sirunyan, A. Tumasyan, W. Adam, E. Aguilo, T. Bergauer, M. Dragicevic, J. Erö, C. Fabjan et al. (CMS Collaboration), Phys. Rev. Lett. 109, 222301 (2012)

[20] J. Hoelck, G. Wolschin, unpublished (2014)

[21] F. Vaccaro, F. Nendzig, G. Wolschin, Europhys. Lett. 102, 42001 (2013)

[22] J. Castillo et al. (ALICE Collaboration), Proc. Quark Matter Conf. Darmstadt, in preparation (2014) 